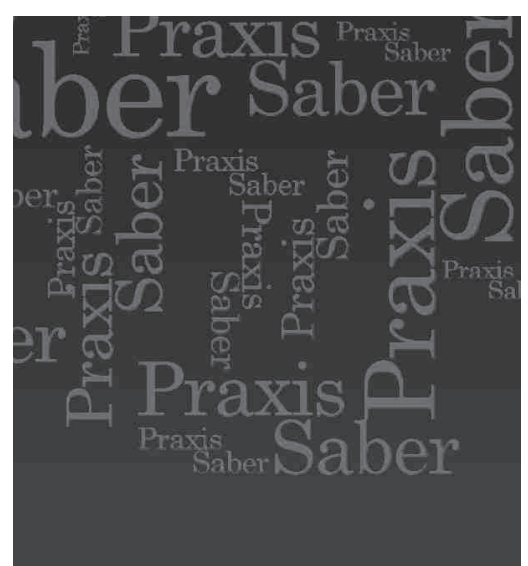

Alberto Pardo Novoa Magíster en Psicología Coordinador de

Investigaciones Programa Licenciatura en Preescolar

Fundación Universitaria

Monserrate, Bogotá

Profesor invitado Maestría en Educación

Universidad Pedagógica y

Tecnológica de Colombia

Grupo de investigación Estilos

Pedagógicos

alberto.pardo@academia.fum.edu.co

Artículo de Reflexión

Recibido: 29 de julio de 2011

Aceptado: 09 de septiembre de 2011

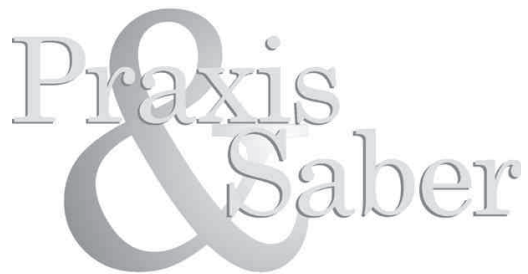

Revista de Investigación y Pedagogía Maestría en Educación. Uptc

\section{VALIDACIÓN Y LEGITIMACIÓN DE LA INVESTIGACIÓN EN EDUCACIÓN Y PEDAGOGÍA ${ }^{1}$}

\section{Resumen}

Desarrolla planteamientos sobre el sentido y los procesos de validación y legitimación de la investigación científicaeneducaciónypedagogía, y plantea criterios y reflexiones que contribuyan al examen de las concepciones y prácticas de investigación comunes en los ámbitos académicos, disciplinares y profesionales. De manera expositiva y argumentada se desarrollan conceptos centrales como ciencia, investigación científica, comunidad de practicantes competentes, validación y legitimación. La exposición de los conceptos destaca el carácter social, público y argumentativo de la ciencia, como sistema, y de la investigación científica, como proceso. Con base en la articulación de esos conceptos, se argumenta y concluye que la comunidad de pares competentes es la idónea, por naturaleza, para validar una investigación, y que la legitimación es un proceso gradual, efectuado por la sociedad desde unos intereses y valores de su transcurrir histórico. A la luz de esos planteamientos, se presentan y cuestionan algunas concepciones y prácticas existentes en nuestros entornos académicos.

Palabras clave: Ciencias de la Educación, Investigación en educación, Investigación pedagógica, Legitimación de la investigación, Validación de la investigación.

1 Este texto, con algunas modificaciones, fue presentado como ponencia en el I Congreso Internacional y II Nacional de Investigación y Pedagogía. Octubre12, 2011. Maestría en Educación, UPTC. Tunja. 


\section{Validation and Authentication of Education and Pedagogical Research}

\section{Summary}

This research paper develops ideas about the meaning and processes of validation and authentication of scientific research in education and pedagogy. It presents criteria and reflections that contribute to the examination of conceptions and common research practices in the academic, disciplinary and professional fields. Core concepts such as science, scientific research, community of competent practitioners, validation and authentication are developed in an explanatory and argued manner. The presentation of the concepts emphasizes the social, public and argumentative nature of science, as a system, and scientific research, as a process. Based on the articulation of these concepts, it is argued, and concluded, that the community of competent peers is the most suitable, by nature, to validate an investigation, and that legitimacy is a gradual process, carried out by society based on limited and historically contingent interests and values. In the light of these approaches, we introduce and challenge some conceptions and existing practices in our academic environments.

Key words: education sciences, research in education, pedagogical research, authentication of the research, validation of the research. Éducation et Pédagogie

\section{Résumé}

On développe des approches sur le sens et les processus de validation et légitimation de la recherche scientifique en éducation et pédagogie, et on expose des critères et des réflexions qui contribuent à l'examen des conceptions et des pratiques de recherche communes dans les milieux académiques, disciplinaires et professionnels. On développe, `a manière d'exposition et d'argumentation, des concepts centraux comme science, recherche scientifique, communauté de praticiens compétents, validation et légitimation. L'exposition des concepts met en évidence le caractère social, publique et argumentatif de la science en tant que système, et de la recherche scientifique en tant que processus. En se basant sur l'articulation 
de ces concepts-là, on argumente et conclut que la communauté de paires compétents est appropriée, par nature, pour valider une recherche, et que la légitimation est un processus graduel, fait par la société `a partir des intérêts et des valeurs de son parcours historique. Il est à la lumière de ces conceptslà, que l'on présente et remet en question quelques conceptions et pratiques existants dans nos environnements académiques.

Mots clés : Sciences de l'Education, Recherche en éducation, Recherche pédagogique, Légitimation de la recherche, Validation de la recherche.

\section{Validação E Legitimação da Pesquisa EM EdUCAÇão e Pedagogia}

\section{Resumo}

O texto desenvolve esboços sobre o sentido e os processos de validação e legitimação da investigação científica na educação e na pedagogia, e delineia critérios e reflexões que contribuem ao exame das concepções e práticas da pesquisa usual nos âmbitos acadêmicos, disciplinares e profissionais. Desenvolvem-se de forma expositiva e argumentativa conceitos centrais como ciência, investigação científica, comunidade de praticantes competentes, validação e legitimação. A exposição dos conceitos salienta o caráter social, público e argumentativo da ciência, como sistema, e da investigação científica, como processo. Baseado na articulação dos conceptos argumenta-se e conclui-se que a comunidade de avaliadores competentes é a idônea, por natureza, para validar uma pesquisa, e que a legitimação é um processo gradual, feito pela sociedade desde uns interesses e valores do seu percurso histórico. À luz dessas explanações, apresentam-se e questionam-se algumas concepções e práticas existentes em nossos ambientes acadêmicos.

Palavras chave: Ciências da Educação, Investigação em educação, Investigação pedagógica, Legitimação da investigação, Validação da investigação.

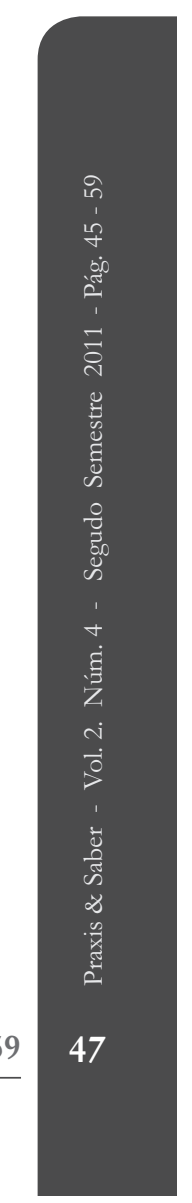




\section{Introducción}

Este artículo busca aportar algunos elementos de orden conceptual y reflexivo a la comprensión crítica y constructiva de las concepciones y prácticas de investigación que circulan en espacios educativos, en particular en la educación superior; estos elementos provienen de una reflexión sistemática sobre diversas experiencias, tanto de acompañamiento a docentes en trabajos de investigación, como de realización conjunta de proyectos investigativos, principalmente, en el ámbito universitario. La exposición se focaliza en la investigación en educación y pedagogía, $\mathrm{y}$ aunque el tema sea relevante para fundamentar una seria labor investigativa, y merezca un tratamiento más amplio y detallado, se desarrollan únicamente dos temas sobre la validación y la legitimación de la investigación, que sirven para sentar bases de ulteriores exámenes y debates académicos:

$1 .^{\circ}$-Visiones sobre validez y legitimidad en ciencia e investigación.

$2 .^{\circ}$-Instancias a las que corresponde la validación y la legitimación de la investigación científica, educativa y pedagógica, y con referencia a qué parámetros.

Más que efectuar un desarrollo secuencial de esos temas, se presentan entrelazados, ya que, de hecho, existen interrelaciones conceptuales y temáticas entre ellos.

\section{Ciencia e investigación científica}

Se propone aquí una manera actual de entender investigación científica y el modo de proceder en ella, y, al mismo tiempo, se examinan algunas de las conceptualizaciones básicas que subyacen en los usos de lenguaje referidos a asuntos de la investigación. Dado que el concepto de ciencia transversaliza y compromete toda la estructura y sentido de las prácticas y producciones investigativas, es necesario asumir una conceptualización estricta de ciencia y de investigación científica, si no se quiere incurrir en un serio déficit que perjudica la producción, el papel y la credibilidad social de esa actividad. Siguiendo a autores como Rosalin Driver, Deanna Kuhn, Gregory Kelly y Yehuda Elkana, se plantea aquí considerar la ciencia como un sistema social de construcción argumentada de conocimiento nuevo y válido, y como un sistema cultural cuyas imágenes de conocimiento están relacionadas con los valores e intereses que 
influyen en los tipos de problemas que se estudian y en los modos de elaborarlos y resolverlos (Driver, 2000; Kelly et al., 1993; Kuhn, 1993; Elkana, 1983); y la investigación científica, en cuanto parte constitutiva de la ciencia, como un proceso o conjunto de operaciones de producción social argumentada de conocimiento nuevo y válido.

Ahora bien, para que una investigación sea de naturaleza científica ha de responder a ciertos criterios y características reconocibles y reconocidos por quienes la practican; tales criterios y cualidades han sido elaborados y acogidos por grupos y comunidades, en el transcurso de procesos histórico-sociales en los que se han venido decantando y transformando, con el aporte de disciplinas como la filosofía, la epistemología, la sociología del conocimiento, la matemática y la lingüística, entre otras. A lo largo de ese devenir fueron emergiendo dos cuestiones cruciales respecto de la naturaleza de la ciencia: una, ¿qué la diferencia del conocimiento común, cotidiano, y dos, al ser diferente a él, ¿cómo tiene presencia en la sociedad? Las respuestas a tales cuestiones han sido, en su orden: los grados de validez del conocimiento construido y la legitimación social de éste.

Hay que tener presente, además, que la ciencia está asociada, aunque no siempre lo ha estado explícitamente, a dos factores indisolublemente ligados entre sí: a una actividad y a las elaboraciones, construcciones o productos de ella.

\section{Validez y validación en ciencia}

Considerada la investigación científica como el conjunto de los procesos de construcción de conocimiento nuevo y válido, se hace preciso definir las cualidades que debe tener para ser calificada como válida. Aunque no ha existido una manera única de asumir la validez de cualquier investigación, hoy ella se entiende relativa a las reglas que estructuran el sistema de construcción de conocimiento; su conceptualización y criterios se han transformado históricamente dentro de los paradigmas; por ello, la validez es sólo una aproximación a la solidez del conocimiento, y solo un posible camino a su fundamentación, señala John Ratcliffe (1983) en su trabajo sobre la noción de validez en la investigación cualitativa.

En la conceptualización esbozada sobre investigación científica se revelan de manera general los criterios para poder ser reconocida como tal; lo cual equivale a que el proceso investigativo cumpla con las condiciones 
de producción acordadas tácita y explícitamente en las prácticas sociales de quienes, con competencia reconocida entre pares, efectúan esa clase de investigación; a saber:

a) El cumplimiento de criterios y normas de producción reconocidos por "la comunidad de practicantes competentes", según el paradigma en el que pretendidamente se inscribe, en un campo o área de conocimiento; es decir, que su sistema argumentativo esté de acuerdo con la racionalidad o lógica de uno de los paradigmas vigentes y dominantes en la actualidad: empírico-analítico, histórico-hermenéutico, críticosocial, o algún otro que pudiera emerger posteriormente.

b) La explicitación y comunicación pública, suficiente y argumentada, del proceso completo de la investigación, o sea, de las posiciones, caminos (metodología) y análisis con los que se establecen los nuevos conocimientos.

La condición de cientificidad de la investigación depende así de su validez. Un proceso investigativo y sus resultados son válidos, es decir, adquieren cualidad de científicos, si cumplen con las condiciones de producción de conocimiento propias del sistema epistemológico y argumentativo en que se basan.

$\mathrm{Si}$, como ya se propuso, se asume una conceptualización de "ciencia" siguiendo la propuesta de autoras como Deanna Kuhn y Rosaline Driver, y se la ajusta a las versiones de los paradigmas, entonces, los temas de la "comunidad que valida" y de la "legitimación" de la investigación científica encuentran en ellos un marco conceptual y de referencia, acreditado en la comunidad internacional, que los articula coherentemente (Driver, 2000; Kuhn, 1993). Por otra parte, este mismo marco favorece un examen crítico y constructivo de nuestras actividades investigativas en educación y pedagogía, y, por lo tanto, puede resultar fecundo para generar trabajos que tengan credibilidad por sí mismos en el entorno nacional e internacional.

La propuesta de Deanna Kuhn, Rosaline Driver y sus seguidores es entender ciencia como un sistema de construcción social argumentada de conocimiento válido, tal como se expuso anteriormente. La referencia a la dimensión social de la investigación científica que introduce esa propuesta no es gratuita; obedece a una manera de entender la naturaleza misma de la cientificidad del conocimiento. Si bien todo conocimiento humano puede pensarse también como de carácter social, al atribuirle la cualidad de científico se le están asignando simultáneamente otros rasgos distintivos, inscritos así mismo en ese ámbito social del conocer. 
Hoy se asumen como propiedades de la cientificidad del conocer humano, construida histórica y culturalmente en Occidente: a) el carácter eminentemente argumentativo y público de esos procesos de construcción; b) la necesidad de un examen público competente, desde las pretensiones de validez paradigmática argumentada, y c) la permanente posibilidad y necesidad de ser sometido a ese examen público y argumentado.

\section{Instancia de validación de la investigación}

Esta dimensión social lleva las consideraciones anteriores a la cuestión de la "comunidad que valida la investigación". Es en el contexto de esa naturaleza social de la ciencia en el que conviene inscribir el asunto de las comunidades competentes implicadas en las funciones sociales de validación y legitimación.

Antes de abordar la pregunta de "cuál es la comunidad que valida", habría que realizar una serie de precisiones conceptuales acerca de qué entender por comunidad; pero para obviar esas disquisiciones, tal vez no pertinentes acá, se sugiere asumir una versión que puede contribuir a precisar el espinoso tema de la validación y legitimación de la producción investigativa científica; con ella, posiblemente, se pueda avanzar en dilucidar puntos álgidos de nuestras prácticas que implican asuntos como la aprobación científico/académica de proyectos y su asignación presupuestal; la aceptación de los trabajos por los comités editoriales de las revistas, y los diferentes usos sociales de esa producción, entre otros.

El concepto de comunidad, en general, aplicable, con algunos ajustes, a los más particulares de comunidad académica, disciplinar, profesional, científica o investigativa, puede ser, como proponen Rafael Ávila y Fernando Vásquez, el de un colectivo de personas que comparten intereses de conocimiento, perspectivas epistemológicas, problemáticas e inquietudes, modos de significar y lenguajes, así como normas de interacción; es decir, la agrupación de personas que comparten escenarios de interlocución, estilos de producción discursiva, criterios de validación, ritos de participación y modos de trabajar en el conocimiento de un campo o área del saber (Ávila, 2004; Vásquez, 2002). Ahora bien, a la luz de esa caracterización, para ser una comunidad no basta con pertenecer a la misma organización, institución o agremiación; es preciso compartir esa cultura como modo de vida o de práctica social. Asumir esas precisiones nos permite adoptar una perspectiva, en cierto sentido, de gran rigor y exigencia cualitativa en cuanto a la dinámica propia de esos colectivos; no se pertenece, pues, a una comunidad por contrato o casualidad; ni por 
compartir espacios y funciones académico-administrativas; se requiere la participación activa en esa vida propia de tales grupos.

La "Comunidad Académica" -siguiendo la propuesta de Ricardo Lucio (1989)-es una instancia que incluye y articula tres ámbitos en el horizonte de la formación: la investigación, la docencia y la proyección social; las clásicas funciones atribuidas como substanciales y definitorias de la universidad. Entonces, Comunidad Académica es, por esencia, la universidad; así, no sería ésta, como tal, la instancia competente para pronunciarse sobre la validez de la investigación, puesto que tiene convocadas en su seno a personas de diferentes disciplinas, profesiones y áreas del conocimiento; solo en la medida que alguna de ellas pertenezca, por su participación activa, a la vida de una comunidad disciplinar, científica o investigativa y cumpla con ciertas cualificaciones particulares, podría ser considerada idónea para desempeñar la función de la validación. Tales cualificaciones consistirían, por una parte, en la actualización, fundamentación y solvencia discursiva sobre el tema o problemática objeto de la investigación, y por otra, solvencia respecto de la racionalidad y metodología paradigmáticas del trabajo para apreciar la validez y argumentar este juicio. Así, un par es representante autorizado de la comunidad o comunidades a las que pertenece, por compartir la misma cultura científica o disciplinar.

Además, la vida de las comunidades verdaderas se manifiesta en otras instancias de la cultura científica; aunque hay actuaciones individuales, se dan enraizadas y articuladas a prácticas sociales, no porque sean siempre efectuadas en compañía de otros, sino porque se llevan a cabo dentro de los parámetros de la naturaleza social, pública y argumentativa de la racionalidad científica, como se expuso antes.

La validación de la cualidad y calidad científica de un trabajo de investigación en educación y pedagogía se va operando paulatina y progresivamente en diferentes instancias socioculturales: siempre por pares, en las comunidades educativas y pedagógicas, por quienes actúan en los comités editoriales de los medios de publicación reconocidos, por quienes presencialmente, en congresos y otros eventos de carácter científico examinan y se pronuncian argumentativamente en público sobre el trabajo en cuestión. Hay que tener presente que la difusión social pública es un camino y condición para que la investigación pueda ser validada.

A propósito de la publicación de la investigación, en medios impresos o por exposición oral, conviene indicar el sentido y función que tienen las referencias bibliográficas respecto de la validación de los productos de 
investigación. Cuando un trabajo particular se encuentra frecuentemente referenciado bajo los cánones de una competente paridad, es signo de que dentro de la cultura científica ha venido obteniendo reconocida validez, al haberse replicado su metodología, o por emplear sus elaboraciones como punto de partida de otros trabajos o como parte de las nuevas construcciones, por nombrar algunas prácticas pertinentes. El solo hecho de que un trabajo sea citado y referenciado no aporta ipso facto a su validación, si no se examina y argumenta su contenido; por ello, los sistemas bibliométricos carecen, en su raíz, de peso como procedimientos de validación y como índices de validez.

A la luz de lo expuesto sobre la validación, para que ésta se vaya realizando es suficiente que los pares, cualitativamente expertos, se vayan pronunciando en diferentes instancias de la cultura científica y en el transcurso de los diversos momentos del proceso social global de la validación; ésta, pues, en sí misma, no consiste en un acto ceremonial de declaración de un juicio o concepto particular. Los actos académicos protocolarios necesarios son sólo eso: actos que aportan a un proceso, a través del cual ellos se van configurando como momentos en los que la validez se va decantando; porque ésta no consiste en un estado o cualidad permanente del conocimiento, sino que puede ser puesta en entredicho e incluso caducar a la luz de los avances que van ocurriendo en el correspondiente campo. La validez científica de un conocimiento es, entonces, una cualidad que se conserva, decae o pierde vigencia a través de los continuos procesos de constatación -según cada paradigma- a que es sometida en el tiempo.

La comunidad que por naturaleza tiene la competencia para validar la investigación no es otra, entonces, que la comunidad de pares, y ella no está circunscrita a instituciones, organizaciones, territorios u otros límites; son sus prácticas sociales, esparcidas, distribuidas y emergentes en diferentes contextos y efectuadas por distintos miembros, las que operan como instancias de validación.

\section{Legitimidad y legitimación en ciencia}

La cuestión de la legitimación de la investigación, a tono con lo expuesto sobre la validación, presenta también un carácter social. Es la sociedad en cuanto tal, en sus diferentes instancias (personas, grupos, organizaciones) y prácticas, la que va asignando sentido, funciones y valores a una producción investigativa circulante en sus entornos. Así, la legitimación de una investigación es un proceso gradual de reconocimiento que la 
sociedad le va otorgando, a través de los usos que de ella se hace y de las valoraciones de todo orden que le atribuye en su vida cultural. Aplicar planteamientos, resultados o metodologías de un trabajo investigativo al tratamiento y solución de situaciones y problemas de la vida sociocultural es estar asignándole a tal trabajo un lugar de reconocimiento; es decir, que ese conocimiento es tenido como elemento genuino de un sector, al menos, de la vida social, y que forma parte de su bagaje cultural.

\section{Instancia de legitimación de la investigación}

La legitimación de la investigación en educación y pedagogía compete, entonces, a la sociedad como tal y, en ella, a sus grupos y demás instancias, como las comunidades académicas, disciplinares, profesionales y científicas, entre otras; ya no solo y estrictamente por criterios de cientificidad, sino por otros intereses y valores del flujo de la vida cultural. Es posible, a la luz de estos planteamientos, que una comunidad disciplinar (o de otro orden), a la vez que va validando científicamente un trabajo investigativo, esté legitimándolo a través de los diversos empleos que de él realiza. Un ciudadano corriente también participa y, en cierto modo, propicia el proceso de legitimación, en la medida en que incorpora a su vida partes sustanciales de trabajos investigativos que conoce o, incluso, desconoce; la presencia de esos aspectos en la vida de los ciudadanos es el signo de la ocurrencia de la legitimación.

Uno de los planteamientos actuales sobre "educación en ciencia" postula precisamente este sentido de la enseñanza en la vida de hoy: contribuir a que el ciudadano corriente esté adecuadamente informado sobre las cuestiones científicas, y disponga de información válida para que sepa tomar decisiones en su vida personal y política, y no quede a merced de otros juegos de poder. Por ello, no se habla de "enseñanza de las ciencias", al menos para el nivel de educación básica y media, sino de "educación en ciencia", como se mencionó. En este sentido se espera que la investigación científicamente válida permee los tejidos de la vida sociocultural y cuente así con su correspondiente legitimidad.

Otro de los retos del conocimiento en educación y pedagogía, además del de la validez, es que responda de manera pertinente y ojalá relevante a las circunstancias, tendencias y necesidades de las instancias sociales; esa pertinencia y esa relevancia pueden estar constituidas con referencia a diversas condiciones, problemáticas, valoraciones e intereses. No sobra advertir, sin embargo, que una investigación no válida científicamente 
puede llegar a ser legitimada, como ocurre con bastante frecuencia, y que un trabajo serio científicamente sea, por otros factores, desconocido, no legitimado. La difusión de los trabajos de investigación -el ser expuestos o referenciados en sus componentes básicos (conceptualizaciones, planteamientos, metodologías, hallazgos)-,es un factor que contribuye a dinamizar el proceso de legitimación social de ellos, en cuanto sus aspectos sustanciales vayan siendo asimilados en las prácticas culturales de los ciudadanos.

Atender a problemáticas de actualidad o a asuntos de interés público general sería una dimensión respecto de la cual la sociedad puede reconocer el valor de las investigaciones. Aunque puede pensarse en la pertinencia y relevancia de los trabajos de investigación como criterios de legitimación, tal como se expuso antes, hay que tener claro que ni la pertinencia ni la relevancia de un trabajo investigativo son criterios de validación.

Para nutrir el examen y la discusión de lo expuesto sobre legitimación de la investigación es pertinente señalar que los valores sociales del conocimiento científico no conviene agotarlos en su utilidad pragmática, en su oportunidad o no para resolver problemas de la realidad, si bien esto es importante. Un conocimiento científicamente válido siempre puede trascender esa dimensión de utilidad y valer por su aporte al enriquecimiento, fortalecimiento o examen de la comprensión del mundo por el hombre y a su enriquecimiento como ser cognoscente integral.

\section{Cuestiones y reflexiones sobre prácticas vigentes}

Las ideas y planteamientos expuestos sirven para develar y examinar sentidos y significados presentes en las subculturas profesionales, académicas y escolares con los que están conformados modos de entender y actuar en relación con la ciencia y la investigación. El empleo reflexivo de esas conceptualizaciones puede contribuir al fortalecimiento del sistema nacional de ciencia, tecnología e innovación, si se adopta una perspectiva crítica y reconstructiva que con responsabilidad social propugne la cualificación de los procesos sociales de construcción de conocimiento válido.

La cuestión de la validez científica de un proceso investigativo y de sus resultados tiene implicaciones serias en muchas de las prácticas vigentes en comunidades académicas, disciplinares y profesionales. 
¿Con qué frecuencia los trabajos realizados y publicados de investigación en educación y en pedagogía cumplen con los criterios presentados aquí?, ¿cuáles fenómenos de índole epistemológica, de intereses de producción de conocimiento, de juegos de poder intelectual, académico o administrativo, por mencionar algunos, existen en el trasfondo de otras prácticas y concepciones de investigación científica y de su validación como tal?

En las experiencias directamente conocidas y estudiadas a través de publicaciones de investigadores expertos, y en otras vividas en formación investigativa de profesores universitarios, que han nutrido estas reflexiones, se identifican a veces fortalezas conforme a los criterios mencionados; existen prácticas de personas y grupos que mantienen en alto un adecuado rigor epistemológico y metodológico, examinan cuidadosamente el trabajo de sus pares y lo discuten con argumentos, a la vez que mantienen como nota dominante en sus posturas una actitud no dogmática, sino siempre susceptible de examen crítico.

También, con frecuencia, en ámbitos profesionales y académicos de formación pre- y posgradual se aprecian vacíos y debilidades especialmente en cuanto a la explicitación epistemológica y metodológica y a la existencia y solidez de la argumentación. Algunas de estas debilidades se presentan más en unos de esos aspectos que en otros, pero infortunadamente existen en todos.

En los trabajos de grado, tanto para pregrado como para maestría, se suele insistir, sin sentido científico, en extensísimos marcos teóricos y en páginas enteras de resultados y conclusiones; mientras que los desarrollos metodológicos suelen carecer de precisión y refinamiento; la metodología, con mucha frecuencia, merece poca atención, según se aprecia en la insuficiente calidad de su presentación. En la publicación de artículos o trabajos investigativos en revistas indexadas o no se repite de alguna manera el mismo sinsentido anterior; pues al exigirse un número reducido de caracteres y páginas -en aras de la síntesis y fácil lectura de los destinatarios- los autores y editores pasan por alto el rigor y profundidad de los aspectos metodológicos y argumentativos. En los eventos llamados de presentación o divulgación no escrita de la producción científica, vuelve a incurrirse en tales despropósitos; se magnifican los resultados y las conclusiones, en desmedro del rigor y fundamentación argumentativa, que deben estar basados en los aspectos epistemológicos y metodológicos de la investigación. 
Pareciera, en resumen, existir un afán por presentar y difundir los logros, sin plantear el camino por medio del cual se llegó a ellos; este recorte impide de hecho que un trabajo de construcción de conocimiento pueda ser validado, es decir, reconocido o no como científico. Una serie de intereses poco científicos parecen subyacer a esas prácticas; a veces se trata de cierto repudio, menosprecio o minimización del papel de los métodos de investigación, sin reconocer que son los procesos y procedimientos metodológicos los garantes de la validez, o sea, de la cientificidad; en otras ocasiones, subyace la propensión a escapar o eludir el rigor, o a disimular su falta; se pretende evitar el riesgo de ser examinado públicamente en los supuestos epistemológicos y en los argumentos en que se fundamentan los resultados. Es como si a los resultados se llegara por arte de magia, a través de trucos invisibles que se deben suponer o adivinar.

En algunas de las prácticas de nuestros entornos educativos -niveles de pre y posgrado- se ha optado por acudir a expertos que emitan juicio por separado: uno para los aspectos temáticos, y otro para los metodológicos. En otras prácticas existe la costumbre de designar o contratar a dos expertos, quienes, independientemente uno del otro, efectúan su pronunciamiento sobre los dos aspectos, pero ocurre con gran frecuencia que esa denominación de "experticia" no cumple con las cualificaciones anteriormente indicadas.

Más aún, sólo con la intención de sembrar inquietudes, se señala aquí la práctica extendida en algunos contextos educativos nacionales de nombrar no lectores, sino "jurados", categoría esta estrictamente medieval, referida al juicio sobre "la verdad" del conocimiento, y no sobre su validez. El empleo de esta categoría en el contexto actual de la ciencia resulta anacrónico y desnaturalizado, puesto que actúa como velo o distractor para ocultar la insuficiencia de ser verdaderos "pares", o, también, para ejercer cierto poder. ¿No resultaría más apropiado, desde todo punto de vista, instaurar definitivamente en su reemplazo el concepto de "par", siempre y cuando éste cumpla con la pertenencia a una comunidad y con las cualificaciones ya indicadas? En investigación en educación y pedagogía, ¿hasta qué punto con esta verdadera "paridad" se podrían superar al menos algunos de los obstáculos inherentes a las prácticas institucionalizadas de actuar como "jurados", que muchas veces proceden dominantemente por criterios administrativos y no por los de cientificidad?

Entre nosotros, por estar atados a ciertas concepciones y prácticas, parece que hemos privilegiado pronunciamientos inmediatistas, pero no 
cualificados, sobre la validez de la investigación, con el interés prioritario de tener y disponer lo más pronto posible de juicios de valor con los cuales proceder burocrática y administrativistamente. Este inmediatismo, la concepción de validez como "producto terminado" y la de validación como un acto $-\mathrm{y}$ no como proceso- han sido factores que han contribuido notablemente a desmejorar el sentido y valor de nuestras prácticas de validación.

Las consideraciones precedentes pueden servir como referentes de apoyo para examinar esas prácticas e ir conformando e instaurando otras con las cuales se propicie la constitución y afianzamiento de verdaderas comunidades científicas.

Los interesados en promover el desarrollo de investigación en educación y pedagogía pueden encontrar provechoso elaborar propuestas, así sean tentativas, a estas cuestiones, $\mathrm{y}$, desde luego, formularse otros interrogantes relativos a estos mismos asuntos.

\section{Conclusión}

Conviene entender la validez de una investigación, fundamentalmente, en relación con su cientificidad; si el trabajo no es explícitamente argumentado desde la racionalidad en que pretende -implícita o explícitamente- estar generado, inscrito; si no es de dominio público, y no es examinado también pública y argumentadamente, entonces carece de credibilidad científica. Cualquier trabajo de investigación -de búsqueda, indagación y construcción de conocimiento-, por sistemático, interesante y pertinente que sea socialmente, si no puede ser sometido a ese riguroso examen argumentado y público no podría considerarse científico. Ahora bien, si esas condiciones de cientificidad no son necesarias, paradójicamente cualquier investigación resultaría válida y habría que prescindir de esta diferenciación.

Por otra parte, la legitimidad de una investigación es cuestión de su vigencia social, de su anidación cultural. El proceso de legitimación es también socio-cultural y paulatino, y de él se encargan, puede decirse que espontáneamente, las diversas instancias de la sociedad. Aunque hay relaciones entre validez y legitimidad, debido a que los procesos de validación y legitimación son por naturaleza socio-culturales, no son, sin embargo, idénticos; pero las comunidades e instancias adecuadas para realizar esas dos funciones pueden en ciertos procesos y momentos coincidir. Los conocimientos validados pueden no tener legitimación 
social, y muchos no validados como científicos pueden estar circulando y conformando tejido socio-cultural.

En el interjuego de esas realidades emergen desafíos significativos que nuestras concepciones y prácticas de investigación en educación y pedagogía tienen que afrontar para proseguir y orientar los esfuerzos de cualificación de la educación.

\section{Referencias}

Ávila, Rafael (2004). La organización institucional del conocimiento, un problema por resolver en la Universidad. Documento inédito. Maestría en Educación. Universidad Pedagógica Nacional. Bogotá.

Driver, Rosaline et al. (2000). "Establishing the norms of scientific argumentation in classrooms". Science Education, 84(3): 287-312.

ElKanA, Yehuda (1983). La ciencia como sistema cultural. Una aproximación antropológica. Boletín Sociedad Colombiana de Epistemología. III, Enero-Dic. 10-11: 65-80.

KeLLY, Gregory et al. (1993). "Science education in sociocultural context: perspectives from the sociology of science". Science Education 77(2): 207-220.

KuHN, Deanna (1993). "Science as argument: implications for teaching and learning scientific thinking". Science Education, 77(3): 319-337.

Lucio, Ricardo (1989). "Educación y pedagogía, enseñanza y didáctica: diferencias y relaciones. Revista de la Universidad de La Salle, Año XI, julio, 17: 35-43.

PÉREZ, Ángel (1995). "La escuela: encrucijada de culturas". Investigación en la Escuela, 26: 7-8.

Ratcliffe, John (1983). Noción de validez en la metodología de la investigación cualitativa. Knowledge: creation, diffusion, utilization, 5(2): 147-167, December. Trad. Gallego, R. et al. 1991. Documento inédito, Maestría en Educación, con énfasis en Docencia Universitaria. Universidad Pedagógica Nacional. Bogotá.

VÁsquez, Fernando (2002). La cultura como texto. Bogotá: Universidad Javeriana. 